\title{
Bicolor Pixels from a Single Active Molecular Material by Surface-Tension-Driven Deposition***
}

\author{
By Ilenia Viola,* Fabio Della Sala, Manuel Piacenza, Laura Favaretto, Massimo Gazzano, Marco Anni, \\ Giovanna Barbarella, Roberto Cingolani, and Giuseppe Gigli*
}

Recently, the supramolecular organization of conjugated molecular systems has received much attention because of the increased application of conjugated materials in complex electronics and optoelectronics devices, such as active matrix displays ${ }^{[1]}$ plastic microelectronics circuits, ${ }^{[2]}$ and large-area panels for lighting, ${ }^{[3-5]}$ requiring high-resolution molecular patterns. The advantages of organic materials as active compounds for these applications lie in the low costs and ease of fabrication, and in the possibility to fine-tune the desired functional properties by minor structural modifications or conformational changes. ${ }^{[6-10]}$ On the other hand, the realization of integrated devices with (sub)micrometer sizes requires the development of advanced lithography techniques suitable for soft materials; the lack of which is often a strong limitation to the use of such materials in practical applications.

Several methods for controlled molecular patterning are currently under investigation, based on either top-down (soft embossing and nanoimprinting ${ }^{[11]}$ ) or bottom-up lithography (spinodal dewetting, ${ }^{[12]}$ micro-contact printing, ${ }^{[13]}$ and surfacetension-driven (STD) techniques ${ }^{[14,15]}$ ). The common goal of these approaches is to dynamically adjust the behavior of molecular solutions at the solid/liquid interface. So far, bottomup lithography looks more promising for patterning conjugated macromolecules. In fact, it allows self-assembly, self-organization, spontaneous aggregation, and recognition capability under suitable modulation by either external or internal stimuli, such as surface-tension gradients, solute diffusion, and molecular weight. ${ }^{[16,17]}$ Unlike the top-down approach, the

[*] Dr. I. Viola, Prof. G. Gigli, Dr. F. Della Sala, Dr. M. Piacenza, Dr. M. Anni, Prof. R. Cingolani

National Nanotechnology Laboratory (NNL) of INFM-CNR

Distretto tecnologico ISUFI

via Arnesano, 73100 Lecce (Italy)

and

Dipartimento di Ingegneria

Università degli Studi di Lecce

via Monteroni, 73100 Lecce (Italy)

E-mail: ilenia.viola@unile.it; giuseppe.gigli@unile.it

L. Favaretto, Dr. M. Gazzano, Dr. G. Barbarella

Istituto per la Sintesi Organica e la Fotoreattività (ISOF)

Area di Ricerca CNR

via Gobetti, 101, 40129 Bologna (Italy)

[※* The authors gratefully acknowledge P. Pompa, S. Lattante, L. Martiradonna, and A. Rizzo (CNR-NNL Lecce, Italy) for PL measurements and useful discussions. This work has been partially funded by MIUR (FIRB RBNE03S7XZ_005 "SYNERGY"). Supporting Information is available online from Wiley InterScience or from the author. bottom-up method is based on the reproduction of a molecular pattern at a characteristic length scale, ${ }^{[18]}$ usually without the need for expensive experimental setups, high-temperature processes, or oxygen exposure, which could damage and alter the physical-chemical properties of the materials. ${ }^{[19]}$ Among the bottom-up techniques, dewetting lithography techniques are attracting growing interest owing to the simplicity of the technological processes, the possibility to realize different pattern resolutions, and the capability to combine and exploit peculiar characteristics of molecular materials, such as self-organization, with dynamical properties of the fluid.

Several works on controlled self-organization during dewetting for engineering of nano- and micrometer patterns in thin liquid films have been reported. ${ }^{[17,20,21]}$ In a recent work, in particular, we demonstrated a lithography technique for the fabrication of monochromatic organic light-emitting diode (OLED) pixel arrays, ${ }^{[14]}$ in which organic materials are patterned with defined features by exploiting the instability phenomena affecting a liquid thin film on a nonwetting surface. ${ }^{[22]}$ Such a method allows us to exploit the organizational capability of molecules, driven by external stimuli, to define well-resolved patterns on large-area substrates.

However, all of the approaches for patterning organic materials in complex structures reported thus far do not allow simultaneous control over the supramolecular organization and the conformation of single molecules, thus strongly limiting the possibility to fully exploit the enormous potential of organic conjugated materials for fine-tuning their electro-optical properties. Such a limitation necessitates the use of more than one active compound with different chemical/physical properties to introduce different functionalities in a device, such as emission at different wavelengths in multicolor displays ${ }^{[23-26]}$ or bipolar conductivity. ${ }^{[27]}$

In this Communication we report on the realization of a bicolor organic micropixel array by a bottom-up dewetting process, exploiting for the first time the conformational flexibility of an oligothiophene derivative, 2,6-bis- $\left(5^{\prime}\right.$-hexyl-

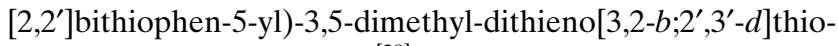
phene (DTT7Me) (Fig. 1). ${ }^{[28]}$ The simultaneous control over both the molecular conformation and the supramolecular pattern of DTT7Me allowed us to vary its emitting properties at micrometer resolution, thereby realizing a well-defined pattern of red- and green-emitting pixels from a single molecular material.

The lithographic approach, that is, STD lithography, ${ }^{[14]}$ is illustrated in Figure 2. STD lithography allows us to control pattern replication by introducing a geometric confinement 


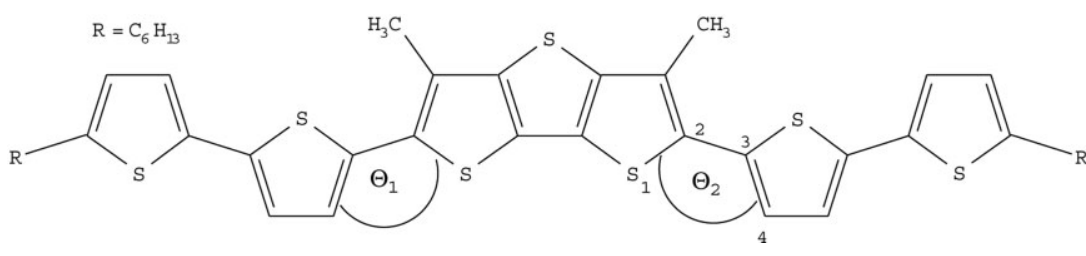

Figure 1. Molecular structure of DTT7Me. Density functional theory (DFT) calculations were carried out with $\mathrm{R}=\mathrm{H}$. The dihedral angles $\theta_{1}$ and $\theta_{2}$ modified in the calculations are shown. a

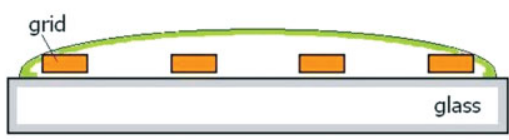

C

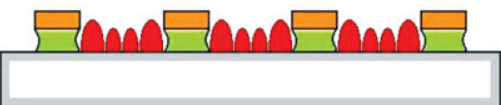

b

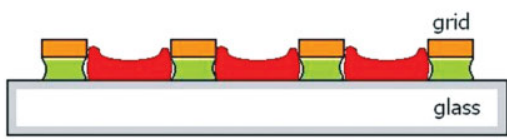

d

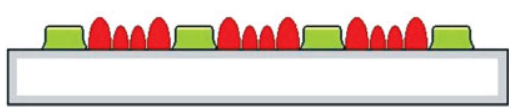

Figure 2. Schematic illustration of the STD procedure, used for patterning a molecular layer of DTT7Me. a) A drop of a dilute solution of DTT7Me in toluene is poured at the center of a template mesh, placed on a glass substrate. b) During solvent evaporation the DTT7Me molecules move both under and inside the mesh. c) The interplay between the energetic conditions at the glass/template and glass/air interfaces induces different molecular arrangements. d) The self-organized pattern, reproduced on the substrate, results in a bicolor pixel structure by a single, active molecular material.

and by modulating the energy parameters of the system (i.e., surface energy) experienced by a drop of dilute solution cast on a substrate.

A geometric confinement placed on the substrate (in our case a micrometer-sized grid template fixed onto a glass substrate) acts as the breaking element during the solvent evaporation. This induces an amplification of the surface instabilities at the liquid interface, driven by polar (hydrogen bonds) and apolar forces (van der Waals and dispersion forces), and by surface-tension gradients (Marangoni-like effects). ${ }^{[29-33]}$ The instabilities will develop with hole-nucleation phenomena and with subsequent outward transport of the material, which confines the dispersed molecules at the edge of the threephase (solid-liquid-vapor) contact line. ${ }^{[17,22]}$ This results in a controlled pattern of molecules that reproduces the positive or negative features of the geometric confinement, depending on the relative surface tensions of the involved materials. $^{[10,14,16]}$

Figure 3 shows a true-color optical image of the patterned structure of DTT7Me obtained from a toluene solution by the STD process. For comparison, an image of a drop-cast DTT7Me film from toluene solution is shown as well. Clear red emission is observed from the drop-cast film, whereas bicolored, pixel-structured red and green emission originates from the STD-patterned film. In particular, green emission is observed from the material underneath the grid bars, whereas red emission is observed from that inside the empty regions.
The optical properties of the STD-patterned film were investigated further by collecting integrated and spatially resolved photoluminescence (PL) spectra by confocal microscopy. Figure 4 displays optical spectra of DTT7Me in solution and in the solid state. Photoluminescence (PL) and absorption in solution show a maximum at 2.47 and $2.92 \mathrm{eV}$, respectively (Fig. 4a). In the STD-patterned film (sample A), the PL spectrum shows a green emission band that is similar to the one in solution, and in addition a band at low energy that is similar to the red emission of the drop-cast film (sample B). Vibronic replicas are also observed in all the spectra.

Spatially resolved spectra, obtained by confocal microscopy (curves (1) and (2) in Fig. 4b), allowed the assignment of the high- and low-energy bands of the patterned film to zones of the sample under the grid and inside the grid mesh, respectively, consistent with the true-color image in Figure 3a. The differences in optical properties between the green and the red pixels of sample A can be explained by considering differing molecular distortions and/or differences in supramolecular packing, which occur during the self-organization driven by the dewetting process. In fact, during nucleation the local variation of the energetic conditions on the DTT7Me solution cast onto the copper $(\mathrm{Cu})$ grid can force individual molecules to assume different molecular conformations, strongly influenced by the spatial features of the pattern. The solvent interface, and thus the solute dispersed in it, experiences an interplay between the energetic conditions at the glass/copper and glass/air interfaces under the template bars and inside the grid holes, respectively, as reported in Table 1 . To further investigate the structural characteristics of the DTT7Me film, and establish a relationship between those characteristics and the optical properties, we carried out atomic force microscopy (AFM) experiments and X-ray analysis on both the patterned and the drop-cast film. AFM measurements on the patterned film (see Fig. 5a and b) reveal an amorphous arrangement of DTT7Me under the bars of the template, whereas well-defined hexagonal crystals are present inside the mesh squares where a different surface energy exists (predominantly owing to the glass surface, see data in Table 1). Conversely, in the AFM topography of the drop-cast film (Fig. $5 \mathrm{c}$ and d) we observe two crystalline phases, probably belonging to different polymorphs. We can easily distinguish both an hexagonal-plate and a rodlike crystalline phase, in agreement with previously observed behavior of DTT7Me powder. ${ }^{[28]}$

These results were confirmed by X-ray diffraction (XRD) measurements performed on both patterned (sample A) and drop-cast films (sample B). The XRD pattern of the pat- 


\section{ADVANCED
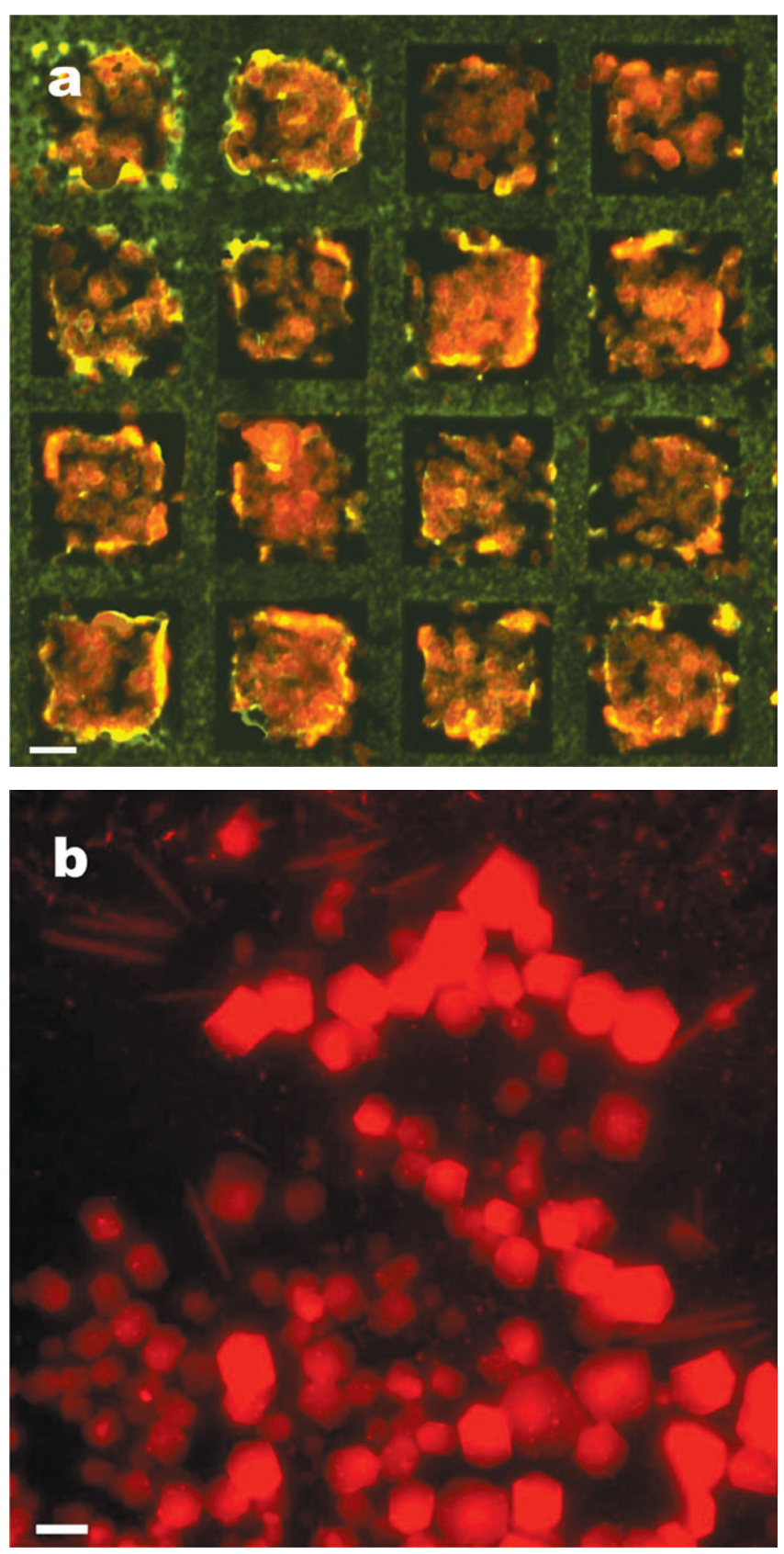

Figure 3. a) Optical image, in true color, of a DTT7Me film patterned by the STD technique (sample A). b) Optical image, in true color, of a dropcast deposition of DTT7Me (sample B). Both crystal types, i.e., hexagonal-plate and rodlike crystals, show red emission. The scale bar in the images corresponds to $10 \mu \mathrm{m}$.

terned film is appreciably different from that of the drop-cast film, as can be seen in Figure 6. In the XRD pattern of DTT7Me cast film (Fig. 6, trace c) several very sharp diffraction peaks appear, revealing a highly ordered and oriented film. In contrast, the XRD profile of the patterned film shows only two appreciable reflections (Fig. 6, trace a) and a broad hump, confirming the simultaneous presence of amorphous material. The lack of any other reflections is a clear indication of the absence of other ordered phases.
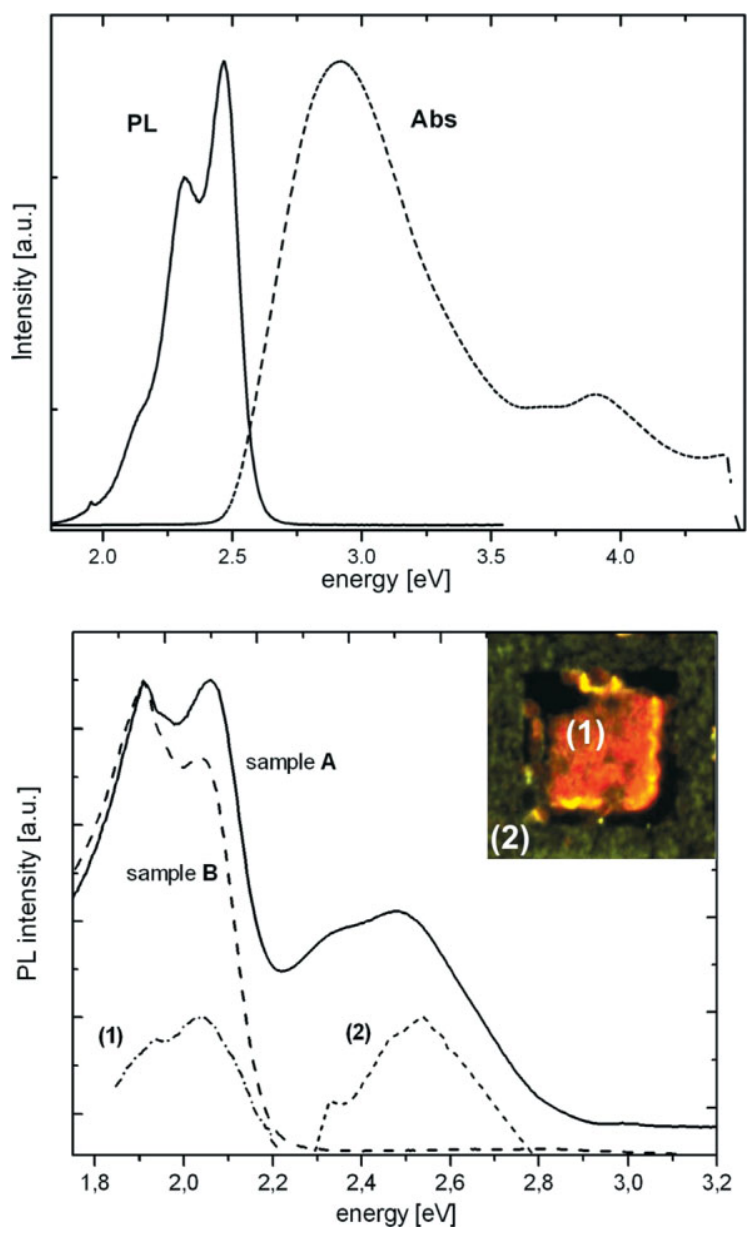

Figure 4. a) PL (solid line) and absorption (dotted line) spectra of DTT7Me in toluene solution. b) PL spectra of DTT7Me in the solid state, deposited by the STD technique (sample A) and by drop-casting (sample B). The bottom part of the graph displays spatially resolved spectra collected by confocal microscopy in zones inside (1) and under (2) the grid mesh of sample $A$, as shown in the inset.

Table 1. Contact angles (CAs) and surface energy of glass and copper substrates.

\begin{tabular}{lccc}
\hline Substrate & $\mathrm{CA} \mathrm{H}_{2} \mathrm{O}$ & $\mathrm{CA} \mathrm{CH}_{2} \mathrm{I}_{2}$ & $\begin{array}{c}\text { Surface energy } \\
{\left[\mathrm{m} \mathrm{N} \mathrm{m}^{-1}\right]}\end{array}$ \\
\hline Glass & $50.0^{\circ} \pm 1.0^{\circ}$ & $36.0^{\circ} \pm 1.4^{\circ}$ & 55.9 \\
Copper & $123.5^{\circ} \pm 1.0^{\circ}$ & $64.0^{\circ} \pm 1.2^{\circ}$ & 27.4 \\
\hline
\end{tabular}

The AFM and XRD results indicate that the molecular solid-state arrangement is strongly connected to the energetic conditions at the liquid/solid interface. More importantly, this characteristic morphology of DTT7Me in the solid state indicates the connection between the supramolecular organization and the emission properties, opening the way to the controlled modulation of the color emission. The photoluminescence properties of substituted oligothiophene crystals are, in particular, strongly related to the single-molecule conformation because of the general low intermolecular interactions 

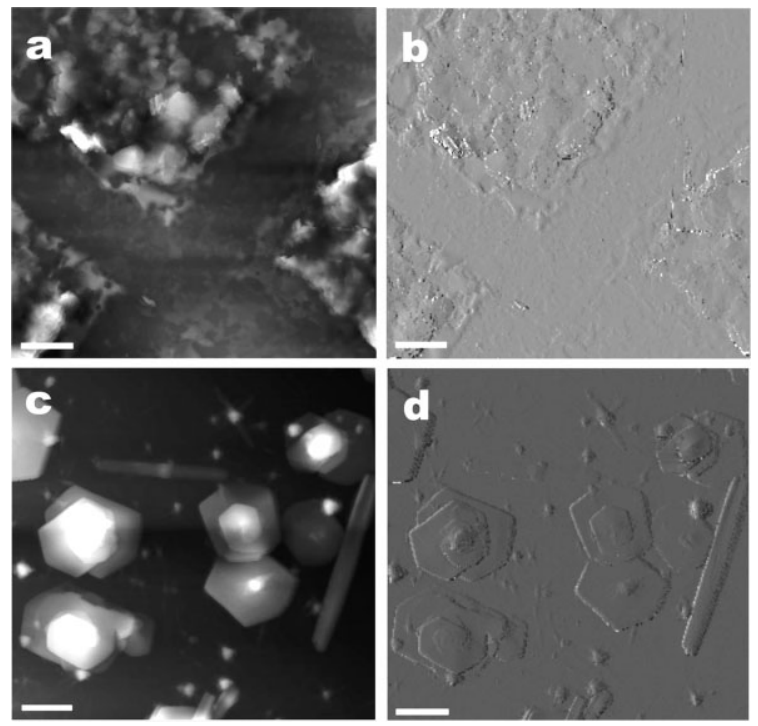

Figure 5. a) AFM topography and b) phase signal of a DTT7Me solution in toluene, deposited on a glass substrate by the STD technique (sample A). Two different self-organization modes can be distinguished, under the template bars and inside the mesh squares, underlining selective differences in surface energy during the STD deposition. c) AFM topography and d) phase signal of a DTT7Me solution in toluene, deposited by drop-casting on a glass substrate (sample B). Microcrystals of different shapes, i.e., hexagonal-plate and rodlike crystals, were observed, suggesting the presence of two polymorphs. The scale bar in the images corresponds to $5 \mu \mathrm{m}$.
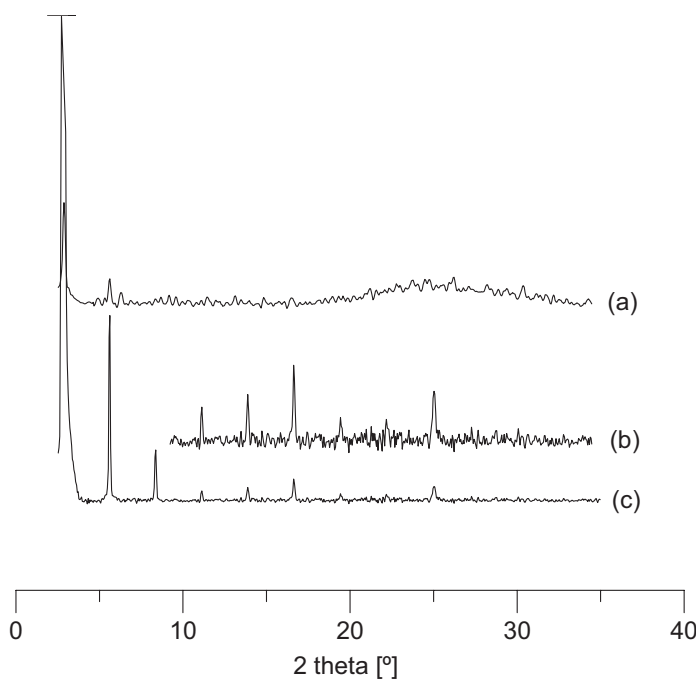

Figure 6. X-ray diffraction profiles of a) the STD-patterned film of DTT7Me and c) the film deposited by drop-casting. Trace (b) is a magnification of (c).

and the ability to strongly deform in the solid state, ${ }^{[34,35]}$ thus preventing the formation of excimers. ${ }^{[25]}$

The variation of the emission energies and its correlation to the molecular distortion have been modeled by first-principles time-dependent density functional theory (TD-DFT) calcula- tions. We restricted our study to a truncated model system, that is, $\mathrm{R}=\mathrm{H}$ in Figure 1.

Geometry optimization of the molecule shows a strongly distorted conformation, owing to the presence of the methyl groups at the central dithienothiophene. The optimized dihedral angle is $\theta_{1}=\theta_{2}=40.5^{\circ}$ (see Fig. 1), and the lowest singlet excitation energy is computed at $2.70 \mathrm{eV}$ with a large (1.76) oscillator strength, in good agreement with the experimental absorption peak (an underestimate by $0.2 \mathrm{eV}$ is typical of TD-DFT calculations in large organic molecules ${ }^{[25]}$ ).

We then consider the rotation along the dihedral angle $\theta_{2}$, fixing $\theta_{1}$ to zero. The computed rotational barriers are smaller than $5 \mathrm{kcal} \mathrm{mol}^{-1}$, meaning that DTT7Me can exist in different conformations in different (solid-state) environments. The lowest calculated excitation energy is $2.43 \mathrm{eV}$ at $0^{\circ}$, where the $\pi$-electron conjugation (at the $\theta_{2}$ position) is complete. At $90^{\circ}$ no $\pi$-electron conjugation occurs, so that the excitation energy reaches the maximum value of $2.81 \mathrm{eV}$. When both $\theta_{1}$ and $\theta_{2}$ are rotated simultaneously, the shifts in excitation energies are roughly doubled. These results show that molecular distortion, that is, inter-ring torsion, can easily (i.e., with a low energetic barrier) lead to a large difference in excitation energies, as is found experimentally.

In the red pixels in particular, molecules are expected to assume a fully planar conformation, as was found in the $\mathrm{X}$-ray analysis of a similar system. ${ }^{[36]}$ In a completely planar system $\left(\theta_{1}=\theta_{2}=0^{\circ}\right)$ the electronic conjugation is enhanced, and the excitation energies are lower than in a structure with rotated substituents.

In the green pixel, the molecules are more distorted and less ordered, as is expected from the amorphous character of the film (Fig. 6a). Thus, molecules are expected to be in, or close to, their ground-state conformation $\left(\theta_{1}=\theta_{2}=40.5^{\circ}\right)$. In this case, the theoretical energy shift between the fully planar and the ground-state structure is $0.27 \mathrm{eV}$, in good agreement with the experimental result of $0.4 \mathrm{eV}$, shown in Figure $4 \mathrm{~b}$.

In conclusion, we report the fabrication of a bicolored pixel structure based on a single luminescent organic molecular material using a surface-energy-driven technique. We succeeded in controlling both the conformation and the supramolecular arrangement of a thiophene-based molecular material (DTT7Me) by exploiting the amplification of the instability phenomena during a dewetting process. The great conformational flexibility of DTT7Me, typical of several substituted oligothiophenes, ${ }^{[37,38]}$ gave us the possibility to achieve the coexistence of two regularly alternating regions on a micrometer scale, emitting light at different wavelengths. The first, a green-light emitting region related to an amorphous phase in which several distorted quasi-equienergetic rotamers were present, was obtained in a region where the geometrical confinement induced low surface energy at the interface. The second, comprising quasi-2D red-light emitting crystals, was obtained where the higher surface energy favored the packing of planar conformations. These results open the way to a new class of self-assembled structures that fully exploit the intrinsic characteristics of conjugated oligomers to finely tune their 
chemical-physical properties through manipulation of their structural features. The proposed single-step, bottom-up technique for the patterning of bicolored arrays demonstrates the feasibility of complex micro- and nanostructures with different functionalities using a single molecular material.

We believe that our approach might be considered a future strategy for the fabrication of a new generation of electronic/ optoelectronic molecular devices and biological detection systems in which recognition events are strongly affected by the interplay between molecular conformation changes and supramolecular organization.

\section{Experimental}

Synthesis: 2,6-Bis-(5'-hexyl-[2,2']bithiophen-5-yl)-3,5-dimethyldithieno[3,2- $\left.b ; 2^{\prime}, 3^{\prime}-d\right]$ thiophene (DTT7Me) was obtained by microwave-assisted cross coupling of 2,6-di-iodo-3,5-dimethyl-

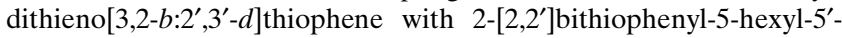
yl(4,4,5,5-tetramethyl)[1,3,2]dioxaborolane, according to details described earlier [28]. The product was recrystallized several times from warm toluene before use. DTT7Me is a liquid crystalline compound, and the first heating and cooling differential scanning calorimetry plots are reported as Supporting Information.

Microstructure Fabrication: Molecular pixel microarrays (sample A) were realized by depositing $5 \mu \mathrm{L}$ of a freshly prepared $0.1 \%$ $(\mathrm{w} / \mathrm{v})$ volatile solution of DTT7Me in warm toluene with a micropipette (Pipet-Lite SL-2 Rainin) on a metallic template fixed on a glass substrate. The substrates were previously cleaned in an ultrasonic bath with three different solvents: acetone, 2-propanol and deionized water (10 min for each step). As a template for geometrical confinement we used a transmission electron microscopy (TEM) calibration copper $(\mathrm{Cu})$ grid with $40 \mu \mathrm{m}$ wide square holes and $20 \mu \mathrm{m}$ wide bars. To check the effect of the geometric confinement on the energetic conditions we characterized a drop-cast film (sample B) obtained from the DTT7Me toluene solution on the same glass substrate.

The surface energies were interpolated by the contact angle goniometer (OCA20, Dataphysics) software by using the polar and dispersion components of contact angles, respectively obtained by the use of water $\left(\mathrm{H}_{2} \mathrm{O}\right)$ and diiodomethane $\left(\mathrm{CH}_{2} \mathrm{I}_{2}\right)$. The morphologies of both types of DTT7Me films, patterned and drop-cast, were characterized by AFM (Solver Pro, NT-MDT) in semi-contact mode.

Optical Characterization: The photoluminescence (PL) spectra were collected by both confocal laser scanning microscopy (FluoView1000, Olympus), for the spatial analysis with a $x y$ resolution of $200 \mathrm{~nm}$, and by spectrophotometry (Eclipse, Varian). The optical images were obtained with a charge-coupled device (CCD) color camera coupled to the confocal microscope.

$X$-Ray Diffraction: XRD patterns were recorded by using the X-ray beam from a copper anode $\left(\lambda_{\text {mean }}=0.15418 \mathrm{~nm}\right)$. The $2 \theta$ interval $\left(2.5^{\circ}-40^{\circ}\right)$ was investigated by using an X'Pert Panalytical diffractometer equipped with an $\mathrm{X}^{\prime}$ Celerator fast detector. The profiles, reported in Figure 6, were collected from the patterned and drop-cast film samples, containing the same amount of material. The XRD pattern of DTT7Me cast film was dominated by the reflection at $2 \theta=2.86^{\circ}$ $(d=3.09 \mathrm{~nm})$. The other reflections belonged to higher orders (up to the 13th order) of the same family of planes. A mean interlayer value of $3.15 \mathrm{~nm}$ was calculated from all the reflections. The XRD profile of the patterned film showed only two significant reflections, corresponding to the first two orders of the same interlayer periodicity of the hexagonal crystals in the drop-cast film. A broad hump in the region $20^{\circ} \leq 2 \theta \leq 30^{\circ}$ confirmed the presence of amorphous material.

Computational Details: We used the B3LYP kernel [39] and the TZVP [40] basis set. Calculations were performed with the
TURBOMOLE [41] program package, using the ESCF [42] module. More details are reported as Supporting Information.

Received: September 2, 2006

Revised: November 15, 2006

Published online: May 23, 2007

[1] H. Sirringhaus, N. Tessler, R. H. Friend, Science 1998, 280, 1741.

[2] G. H. Gelinck, H. E. A. Huitema, E. van Veenendaal, E. Cantatore, L. Schrijnemakers, J. B. P. H. van der Putten, T. C. T. Geuns, M. Beenhakkers, J. B. Giesbers, B. H. Huisman, E. J. Meijer, E. M. Benito, F. J. Touwslager, A. W. Marsman, B. J. E. van Rens, D. M. de Leeuw, Nat. Mater. 2004, 3, 106.

[3] J. H. Burroughes, D. D. C. Bradley, A. R. Brown, R. N. Marks, K. Mackay, R. H. Friend, P. L. Burns, A. B. Holmes, Nature 1990, 347, 539.

[4] T. R. Hebner, C. C. Wu, D. Marcy, M. H. Lu, J. C. Sturm, Appl. Phys. Lett. 1998, 72, 519.

[5] H. Sirringhaus, P. J. Brown, R. H. Friend, M. M. Nielsen, K. Bechgaard, B. M. W. Langeveld-Voss, A. J. H. Spiering, R. A. J. Janssen, E. W. Meijer, P. Herwig, D. M. de Leeuw, Nature 1999, 401, 685.

[6] G. Gigli, M. Anni, R. Cingolani, G. Barbarella, Advanced Semiconductor and Organic Nano-Techniques (Ed: H. Morkoc), Elsevier, New York, NY 2003, Ch. 5, pp. 241-291.

[7] G. Barbarella, M. Melucci, G. Sotgiu, Adv. Mater. 2005, 17, 1581.

[8] D. Grebner, M. Helbig, S. Rentsch, J. Phys. Chem. 1995, 99, 1699.

[9] O. Karthaus, C. Adachi, S. Kurimura, T. Oyamada, Appl. Phys. Lett. 2004, 84, 4697.

[10] M. Cavallini, R. Lazzaroni, R. Zamboni, F. Biscarini, D. Timpel, F. Zerbetto, G. Clarkson, D. Leigh, J. Phys. Chem. B 2001, 105, 10826.

[11] Y. Xia, G. M. Whitesides, Angew. Chem. Int. Ed. 1998, 37, 550.

[12] A. Sharma, Langmuir 1993, 9, 861.

[13] X. M. Zhao, Y. Xia, G. M. Whitesides, J. Mater. Chem. 1997, 7, 1069.

[14] I. Viola, M. Mazzeo, A. Passabì, S. D'Amone, R. Cingolani, G. Gigli, Adv. Mater. 2005, 17, 2935.

[15] M. Cavallini, F. Biscarini, M. Massi, A. Farran-Morales, D. A. Leigh, F. Zerbetto, Nano Lett. 2002, 2, 635.

[16] J. Zhao, S. Jiang, Q. Wang, X. Liu, X. Ji, B. Jiang, Appl. Surf. Sci. 2004, 236, 131.

[17] R. D. Deegan, O. Bakajin, T. F. Dupont, G. Huber, S. R. Nagel, T. A. Witten, Nature 1997, 389, 827.

[18] C. Bowmann, A. C. Newell, Rev. Mod. Phys. 1998, 70, 289.

[19] H. C. Scheer, H. Schulz, T. Hoffmann, C. M. S. Torres, J. Vac. Sci. Technol. B 1998, 16, 3917.

[20] O. Karthaus, T. Koito, M. Shimomura, Mater. Sci. Eng. C 1999, 8-9, 523.

[21] S. Harkema, E. Schäffer, M. D. Morariu, U. Steiner, Langmuir 2003, 19, 9714.

[22] M. Maillard, L. Motte, M. P. Pileni, Adv. Mater. 2001, 13, 200.

[23] M. Granström, O. Inganäs, Appl. Phys. Lett. 1996, 68, 147.

[24] G. Gigli, O. Inganäs, M. Anni, M. De Vittorio, R. Cingolani, G. Barbarella, L. Favaretto, Appl. Phys. Lett. 2001, 78, 1493.

[25] M. Mazzeo, V. Vitale, F. Della Sala, M. Anni, G. Barbarella, L. Favaretto, G. Sotgiu, R. Cingolani, G. Gigli, Adv. Mater. 2005, 17, 34.

[26] G. Li, J. Shinar, Appl. Phys. Lett. 2003, 83, 5359.

[27] Y. Hatanaka, M. Niraula, A. Nakamura, T. Aoki, Appl. Surf. Sci. 2001, 175-176, 462 .

[28] F. Cicoira, C. Santato, M. Melucci, L. Favaretto, M. Gazzano, M. Muccini, G. Barbarella, Adv. Mater. 2006, 18, 169.

[29] K. Kargupta, A. Sharma, Langmuir 2002, 18, 1893.

[30] M. Massi, M. Cavallini, S. Stagni, A. Palazzi, F. Biscarini, Mater. Sci. Eng. C 2003, 23, 923.

[31] K. Y. Suh, H. H. Lee, J. Chem. Phys. 2001, 115, 8204. 
[32] K. Y. Suh, J. Park, H. H. Lee, J. Chem. Phys. 2002, 116, 7714.

[33] X. Wang, M. Östblom, T. Johansson, O. Inganäs, Thin Solid Films 2004, 449, 125.

[34] G. Gigli, F. Della Sala, M. Lomascolo, M. Anni, G. Barbarella, A. Di Carlo, P. Lugli, R. Cingolani, Phys. Rev. Lett. 2001, 86, 167.

[35] E. Tedesco, F. Della Sala, L. Favaretto, G. Barbarella, D. AlbesaJove, D. Pisignano, G. Gigli, R. Cingolani, K. D. M. Harris, J. Am. Chem. Soc. 2003, 125, 12277.

[36] M. Gazzano, X-ray analysis of 2,6-Bis-(5'-hexyl-[2,2']thiophen-5-yl)3,5-dithieno[3,2-b;2, $\left.3^{\prime}-d\right]$ thiophene, unpublished.
[37] G. Gigli, R. Rinaldi, M. Lomascolo, R. Cingolani, G. Barbarella, M. Zambianchi, Appl. Phys. Lett. 1998, 72, 1013.

[38] G. Barbarella, M. Zambianchi, L. Antolini, P. Ostoja, P. Maccagnani, A. Bongini, E. A. Marseglia, E. Tedesco, G. Gigli, R. Cingolani, J. Am. Chem. Soc. 1999, 121, 8920.

[39] A. D. Becke, J. Chem. Phys. 1993, 98, 5648.

[40] A. Schäfer, C. Huber, R. Ahlrichs, J. Chem. Phys. 1994, 100, 5829.

[41] R. Ahlrichs, M. Bär, M. Häser, H. Horn, C. Kölmel, Chem. Phys. Lett. 1999, 162, 165.

[42] R. Bauernschmitt, R. Ahlrichs, J. Chem. Phys. 1996, 104, 9047. 